\title{
Asteroids and their collisional disruption
}

\author{
Akiko M. Nakamura ${ }^{1}$ and Patrick Michel ${ }^{2}$ \\ 1 Graduate School of Science, Kobe University, 1-1 Rokkodai-cho, Nada-ku, Kobe, \\ Japan amnakamu@kobe-u.ac.jp \\ 2 Laboratoire Cassiopée, Observatoire de la Côte d'Azur, CNRS, Université de \\ Nice Sophia-Antipolis, boulevard de l'Observatoire, 06300 Nice, France \\ michel@oca.eu
}

\begin{abstract}
The collisional process between small bodies is one of the key processes in the formation and evolution of a planetary system. Asteroids are the remnants which kept the memory of collisional processes that took place in different regions of our Solar System during its past and present history. Telescopic observations have collected data of such records, such as the ones provided by asteroid dynamical families and their related dust bands. Meteorites, micrometeorites, and interplanetary dust particles (IDPs), which are pieces of asteroids or comets collected on Earth, provide also information on the material properties of these small bodies, although they may only tell us about the strongest components capable of surviving the entry in Earth's atmosphere. In order to understand the collisional process, impact experiments have been performed in laboratory, using as targets terrestrial rocks whose mechanical properties are similar to those of some meteorites. The results of experiments together with numerical simulations and theoretical considerations have led to the conclusion that most asteroids smaller than several tens of kilometers in size have experienced major impact events, during which they have been at least severely shattered so that cracks and voids could be formed in their interior. For those who underwent a catastrophic disruption as a result of a collision at high impact energy, the outcome has been the formation of an asteroid family, some of which are still identifiable in the main asteroid belt. During such an event, the largest fragments that originate from the parent body can be large enough to undergo gravitational re-accumulations, so that at the end of the process, the cluster of fragments larger than a few hundreds of meters resulting from such a disruption is mostly composed of gravitational aggregates or rubble-piles. Spacecraft explorations of multi-kilometer asteroids, namely 951 Gaspra, $243 \mathrm{Ida}$, and 433 Eros, who belong to the S taxonomic class - the dominant class in the inner Solar System - revealed that the surface of these bodies are shaped by impact processes, and that there bulk density (2.6 and $2.67 \mathrm{~g} / \mathrm{cm}^{3}$ for Ida and Eros, respectively) is generally
\end{abstract}

To be published in: "Small Bodies in Planetary Systems". (Mann, Nakamura, Mukai eds.) Submitted to Lecture Notes in Physics series by Springer Funded by the 21the Century COE Program "Origin and Evolution of Planetary Systems" 
lower than the supposed grain density of their material. However, direct evidence of a rubble-pile structure has not been obtained, as the only information on their internal structure are inferred mostly from their surface properties. Conversely, in spite of its small self-gravity, the sub-kilometer asteroid 25143 Itokawa explored by the JAXA Hayabusa spacecraft in 2005 is the first S-class asteroid whose porosity is estimated to be as high as $40 \%$ (with a bulk density of $1.9 \mathrm{~g} / \mathrm{cm}^{3}$ ) and thus is considered to be a gravitational aggregate formed by reaccumulation of smaller pieces. The boulders on Itokawa have shapes and structures similar to those of laboratory rock fragments, suggesting some universal character of the disruption process. Since more and more asteroids are believed to have substantial porosity, current studies on the collisional disruption of solid bodies are to be extended to porous bodies, taking into account microporisity effects which have been neglected so far. Such porous bodies are not only present in the asteroid populations (Near-Earth Objects, main belt and Trojan asteroids) but they are also supposed to constitute the populations evolving in the outer Solar System (Kuiper belt objects) and beyond (long-period comets). Thus, understanding the collisional process for different kinds of material appears crucial to determine its influence in the history of different populations of small bodies.

\section{Introduction}

Since the end of the accretion phase of the Solar System which led to the formation of our planets approximately $4.5 \mathrm{Gyr}$ ago, the outcomes of mutual direct collisions between small bodies have mostly been disruptive and played a major role in the formation, evolution and shape of small body populations. The major collisional disruption zones in the Solar System are the main asteroid belt ([16]), located between the orbits of Mars and Jupiter, and the Edgeworth-Kuiper belt ([18]) beyond Neptune's orbit. Mutual collisional velocities are typically of the order of several $\mathrm{km} / \mathrm{s}$ in the main asteroid belt ([6]), whereas they are about $1 \mathrm{~km} / \mathrm{s}$ or less in the Kuiper belt (e.g., [18]). The collisional process is very complex and is still poorly understood, despite recent progresses. Thus, it is a major area of research which is studied by three interrelated and complimentary approaches, namely, laboratory experiments, numerical modeling and scaling theories ([28]). Observations of small bodies, both ground-based and space-based, provide constraints to these studies and evidence that the collisional process is still active. Asteroids have been the targets of ground-based telescopic observations for more than two centuries ([21]) and have become more recently the targets of space missions (fly-by, in-situ and sample return). The observational data of asteroids are therefore more and more abundant and have statistical significance in many aspects. Additionally, we have the very samples from asteroids, i.e. meteorites, for in-depth microscopic analyses. Therefore, the asteroids are so far the most

To be published in: "Small Bodies in Planetary Systems". (Mann, Nakamura, Mukai eds.) 
studied objects in terms of physical properties connected to the collisional process.

In this chapter, a brief introduction to the current state of our knowledge on asteroids is first exposed, allowing further reading of more detailed reviews in recent literatures (e.g., [8]). Emphases are put on the evidence and processes related to collisional disruption events. Basic equations and quantities that are used to describe high-velocity collisions between solid bodies are then given and the impact response of solid bodies is illustrated by addressing the laboratory analogue experiments. Finally, evidence of impact processes found on the small asteroid 25143 Itokawa explored by the JAXA (Japan Aerospace Exploration Agency) Hayabusa spacecraft are introduced; open problems and perspectives are then indicated.

\section{Asteroids}

\subsection{Orbital and Size Distribution}

Asteroids are small rocky bodies whose large majority do not have any signature of volatile activities. Only very recently, a few of them have been observed to have some sporadic activities ([30]). Most of these bodies are orbiting between Mars and Jupiter in a region called the Main Belt (MB hereafter). The structure of the MB is dynamically constrained by the gravitational perturbations of planets, and more particularly the biggest planet Jupiter. Mean motion resonances with Jupiter, which occur at locations where the orbital period of a small body is proportional to that of Jupiter, correspond to empty zones in the MB called Kirkwood gaps, after the name of their discoverer. Such gaps are a signature of the efficiency of such resonances to destabilize the trajectory of small bodies located into them. Fig. 1 shows the distribution of semi-major axis and inclination of the bodies in the MB. The gaps are visible and are associated to those dynamical mechanisms such as mean motion resonances with Jupiter, and secular resonances which occur when the frequency of the longitude of perihelion of a small body is equal to one of the proper frequencies of the Solar System generally associated to the average frequency of the longitude of perihelion of a planet (such as the $\nu_{6}$ secular resonance associated to Saturn). Asteroids located in those resonances are rapidly transported from the MB to the Near-Earth space due to the fast increase of their eccentricity caused by the resonant dynamics. Thus, the $\nu_{6}$ secular resonance at the inner edge of the MB and the $3: 1$ mean motion resonance with Jupiter at 2.5 AU are among the most effective resonances that supply asteroids to the near Earth object (NEO) population. The timescale for increasing the eccentricity from MB value to Earth-crossing orbits is only a few Myr in these resonances ([24], [43]). The median lifetime of the NEO population is about $9 \mathrm{Myr}([25])$, and the end-state is either a collision with the Sun for $60 \%$ of them, an ejection outside Jupiter's orbit for $30 \%$ of them, 
or a collision with a planet for the rest of the population. However, this population has been kept more or less in a steady-state number over more than 3 Byr ([36]), as indicated by the dating and counting of Moon's craters, which suggest that the flux of impactors has been kept constant on average (apart from some short fluctuations) over this period. This is due to the fact that while some NEOs reach their end-state, collisions occur in the MB. These collisions generate new fragments whose evolutions lead them to a resonance and eventually to the Near-Earth space.

There are also populations of small bodies that lie farther away from the Sun than the MB. The Trojan asteroids evolve in the 1: 1 mean motion resonance with Jupiter on the same orbit as the planet, $60^{\circ}$ before and after the planet on the Lagrangian points $L_{4}$ and $L_{5}$ of the three-body problem. Small bodies with perihelia greater than Jupiter's semimajor axis (5.2 AU) and semimajor axes smaller than that of Neptune (30.1 AU) are called Centaurs. These objects originally come from the population of trans-Neptunian objects (TNOs), also called Kuiper belt objects (KBOs), located beyond Neptune's orbit. In all these populations, small bodies with well-determined orbits have been given a permanent number and a name, such as the small NEO 25143 Itokawa, the largest asteroid in the MB called 1 Ceres, the Centaur 5145 Pholus, and the first discovered TNO (15760) $1992 \mathrm{QB}_{1}$.

The clusters of asteroids that are easily identified in the proper orbital element space in the MB are dynamical asteroid families. Proper elements are quasi-integrals of motion which are more stable than the osculating elements, although some mechanisms such as the Yarkovsky thermal effect can cause slow variations over time. In such a proper element space, the points related to real objects have a stronger link to their original place than in the osculating element space, which allows the identification of connected groups related to asteroid families. In Fig. 1, three prominent asteroid families, Themis, Eos, and Koronis families that were first discovered in 1918 ([33]) are indicated. The origin of an asteroid family is the catastrophic disruption of a large asteroid (called the parent body of the family) as a result of a collision with a smaller body. The largest children formed during such events, by reaccumulation of small fragments of the parent body are the known family members (see Michel's chapter in this issue), which have been identified thanks to their large enough size, spectra similarities and small dispersion by ground-base observations. The finest portion of the ejecta from the parent body spreads further and sometimes can be identified as dust bands associated to the family (see Ishiguro and Ueno's chapter in this issue). The age of a young asteroid family is determined by directly tracking the orbital evolutions of the family members backwards in time all the way to their starting orbits, provided that the time since the birth event is not long enough to reach the time of unpredictability of the orbital motions due to chaos and highly non-linear phenomena. The Karin cluster is such a young group of asteroids produced by the disruption of $\mathrm{a} \approx 30 \mathrm{~km}$-size body, only $5.75 \pm 0.2 \mathrm{Myr}$ ago ([51]). Because the proper orbital elements of family members undergo some diffusion due to high-order 


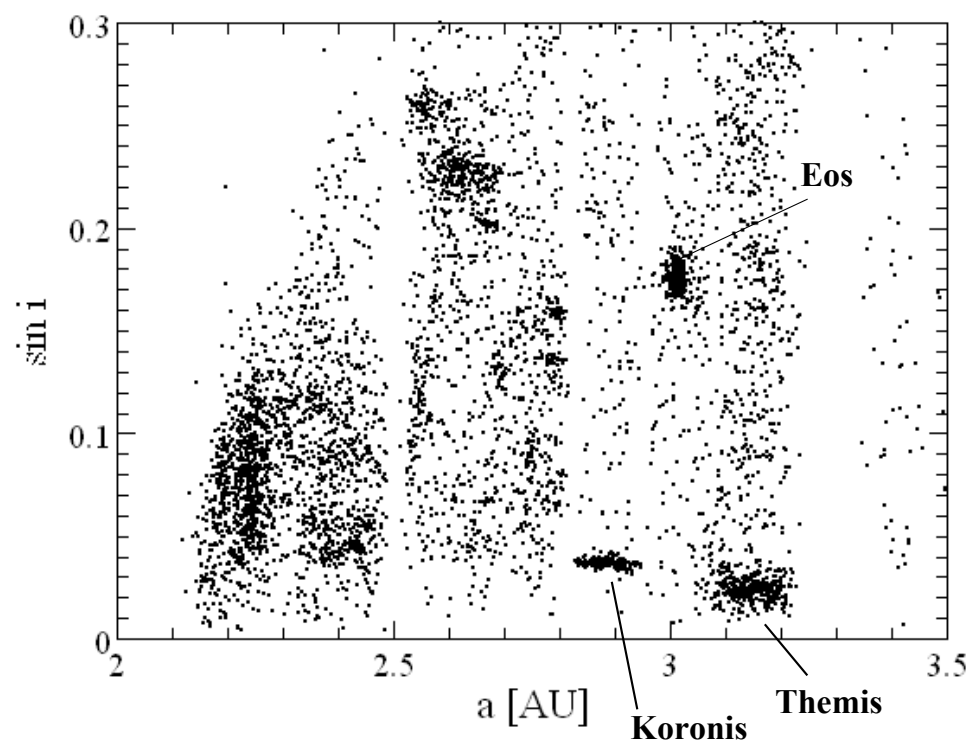

Fig. 1. The distribution of 5335 numbered asteroids with respect to proper semimajor axis $a$ and inclination $i$ (see ([41]); data from PDS Small Body Node). The inner edge is determined by the $\nu_{6}$ secular resonance. The gap at $2.5 \mathrm{AU}$ corresponds to the location of the $3: 1$ mean motion resonance with Jupiter. Themis, Eos, Koronis asteroid families are indicated. See also Fig.14 in Jewitt's chapter in this issue.

resonances and the Yarkovsky effect, which leads to a semimajor axis drift depending on the object's spin, orbit, and material properties ([8]), it is, as we just said, impossible to determine the age of old families by direct orbital integration backward in time based on purely dynamical considerations.

The number of asteroids increases with the absolute magnitude $H$. The amount of the reflected sunlight from an asteroid is proportional to the square of the diameter; the absolute magnitude $H$ and the diameter $D$ of an object are related by the following expression:

$$
D=\frac{1329}{\sqrt{p_{v}}} 10^{-\frac{H}{5}}
$$

where $p_{v}$ is the geometric albedo, whose value ranges from 0.04 and 0.4 in the different taxonomic classes of the asteroid population. Thus, when the albedo is not known, the diameter of an asteroid can roughly be estimated with an ambiguity within a factor of a few. The cumulative size distribution of asteroids having a diameter larger than a given diameter $D$, called $N(>$ $D$ ), increases with decreasing $D$. A power-law is generally used to fit this distribution with a power of about -2 in the range $1-10^{3} \mathrm{~km}$ (see also Sec. 2.4), although the slope is still uncertain in the kilometer-size range. As for asteroid 
families, power-law exponents mostly in the range -2 to -4 are found for the size distribution of large members ([70]).

\subsection{Asteriod composition}

The composition of asteroids is continuously investigated by remote-sensing techniques, such as spectroscopy of the reflected sunlight from the surface, and in laboratory by the analyses of meteorites. Establishing the link between asteroids and meteorites is crucial as it can provide clues on the composition and internal structure of asteroids and on the orbital properties of the meteorites' parent bodies. Moreover, an identified link would give answers to the question of where in the Solar System those meteorites experienced the different chemical and physical processes.

The dynamical link between asteroids and meteorites can be studied both by model calculations of the orbital evolutions of main belt asteroids and by observationally determined orbits of meteorite falls ([60]). Material connection between asteroids and meteorites can be estimated from the visible and near-infrared (NIR) spectroscopic ground-based observations of asteroids and $\mathrm{X}$-ray and gamma-ray spectroscopic observations made in-situ by spacecrafts visiting an asteroid. The diagnostic features of silicates in the visible and NIR reflectance spectra are two relatively narrow and symmetric absorption features of pyroxene (at wavelengths of 0.9 and $1.9 \mu \mathrm{m}$ ) and a broad asymmetric $1 \mu \mathrm{m}$ absorption feature of olivine. Different types of meteorites have been related to different taxonomic classes of asteroids by a resemblance in color and albedo. The taxonomic classification of asteroids has been extended from the original one made in the 20th century. Asteroids were initially classified into $\mathrm{C}$ and S (i.e., carbonaceous and stony)-classes ([13]), and then into several classes, including M (metal) -class, but because some subtle differences have been identified within each class, those initial classes have been split into sub-classes ([12]). Then, new classes have been defined, such as, for instance the W-class, which is composed of M-class asteroids for which a $3 \mu \mathrm{m}$ water absorption band has been found in their spectra ([58]).

The most common taxonomic class in the inner MB is the S-class. S-class asteroids typically have a geometric albedo of $\sim 0.15([62])$ and the meteorites believed to be associated to this class are the ordinary chondrites, the most abundant meteorites collected on Earth's surface. However, until recently, it was not clear whether S-class asteroids are really the parent bodies of ordinary chondrites, because a detailed comparison of the reflectance spectra shows a mismatch in the spectral slope, albedo, and absorption band depth: the spectra of S-class asteroids are redder and darker than those of ordinary chondrites and the NIR silicate absorption bands are shallower ([15]). This discrepancy has then been understood as an effect of the space weathering which affects the top thin surface of small bodies. Surface modification processes due to the space environment, such as solar wind ion implantation, sputtering, and micrometeorite bombardment and their resulting optical effects all correspond to

To be published in: "Small Bodies in Planetary Systems". (Mann, Nakamura, Mukai eds.) 
what is called space weathering. Indeed, X-ray fluorescence spectroscopy performed on the asteroids 433 Eros and 25143 Itokawa shows that these bodies have elemental compositions consistent with that of ordinary chondrites ([63], [53]).

C-class asteroids have low geometric albedo, typically $\sim 0.05$, and monotonic spectra in the visible wavelength range with ultraviolet absorption feature. They are believed to be the parent bodies of carbonaceous chondrites and are abundant in the middle zone of the MB. Darker and redder colored asteroids, which are considered to have more organics, are dominant in the outer region of the MB and among Trojan asteroids (called D-class asteroids). The orbital distribution of the different asteroid classes, which shows some groupings, is an indication that the degree of thermal metamorphism and consequently the aqueous alteration depends on the heliocentric distance from the Sun.

\subsection{Internal Structure}

Mass estimates of asteroids were very limited until the beginning of spacecraft explorations towards asteroids and the precise determination of asteroid orbits. The masses of the largest ones have been determined from mutual close approaches and by their gravitational perturbations onto smaller objects. Moreover, the masses of visited asteroids could also be determined by their perturbation on the observing spacescraft. Then, the discovery by the Galileo spacescraft ([14]) of a satellite, called Dactyl, around the asteroid 243 Ida stimulated search efforts for binary systems. Now photometric light-curve observations, radar-imaging observations, and direct imaging using adaptive optics attached on the largest telescopes are used to detect binary systems. Once the asteroid mass is determined, the bulk density can be calculated using a shape model.

The current internal structure of asteroids is a consequence of their collisional history. It can range from monolithic, fractured and shattered due to moderate impacts to gravitationally reagglomerated rubble piles. The bulk density of an asteroid gives a first-order indication of its internal structure. For instance, the C-class asteroid 253 Mathilde visited by the NEAR spacecraft in 1997 has a bulk density of $1.3 \mathrm{~g} / \mathrm{cm}^{3}$ ([68]). This is much lower than the bulk density of carbonaceous chondrites. For example, CI and CM chondrites have average bulk densities of 2.1 and $2.2 \mathrm{~g} / \mathrm{cm}^{3}$, whereas the average bulk densities of $\mathrm{CR}, \mathrm{CO}$, and $\mathrm{CV}$ chondrites are greater than $3 \mathrm{~g} / \mathrm{cm}^{3}$ ([10]). The low bulk density of Mathilde is interpreted as an indication of high porosity inside the body. Note that the type of porosity measured in meteorites is microporosity, which is related to fractures, voids, and pores on the scale of tens of micrometers. Based on their grain density of CM chondrites $\left(\sim 2.7 \mathrm{~g} / \mathrm{cm}^{3}\right)$, $\mathrm{CM}$ chondrites have a degree of microporosity of about $12 \%$. If Mathilde has material components and microstructures similar to $\mathrm{CM}$ chondrites, its total porosity is $\sim 52 \%$ and its macroporosity accounts for $\sim 40 \%$. The typical

To be published in: "Small Bodies in Planetary Systems". (Mann, Nakamura, Mukai eds.) 
macroporosity of S-class asteroids is about $20 \%$, based on the bulk density measured for Ida and Eros, 2.6 and $2.67 \mathrm{~g} / \mathrm{cm}^{3}$, respectively, although the 500 meter S-class asteroid 25413 Itokawa, with a bulk density of $1.9 \mathrm{~g} / \mathrm{cm}^{3}$, has a macroporosity of about $40 \%$, similar to the one of the C-class Mathilde. This shows the great diversity of asteroids' properties, even in a same spectral class.

These information about the internal structure are extremely important. The outcome of a collisional disruption of an asteroid depends highly on its mechanical properties ([28], [56], [39]) which constrain the transmission efficiency of the impact energy throughout the body. For instance, the presence of five big craters larger than $5 \mathrm{~km}$ in diameter on the surface of the asteroid Mathilde is considered as an indication that porous asteroids are more robust against impacts (absorb more greatly the impact energy). Indeed, based on our current understanding, a non-porous object of similar size would not have survived the impact events needed to create such craters. Actually, the transfer of the shock wave throughout the body, more efficient in a non porous material, would have destroyed it all.

As one can see, the information that we have on the internal structure of small bodies are all indirect. Thus, only in-situ investigations by space missions will allow us to have a more direct characterization. However, even in this case, challenging techniques will have to be used, such as radar tomography, to characterize the properties deep inside an object.

\subsection{Timescales of processes during asteroid evolution histories}

The evolution history of asteroids can be illustrated by key timescales. The collision lifetime $\tau_{c o l}$ of an asteroid of diameter $D$ has been estimated assuming constant collisional velocities and impact rates over time, i.e. assuming the orbital structure of asteroids does not change much during the population history. Its expression is given by:

$$
\frac{1}{\tau_{c o l}(D)}=\int_{D_{p}^{*}} \frac{P_{i}}{\pi} \sigma_{c o l} n\left(D^{*}\right) \mathrm{d} D^{*}
$$

where $D_{p}^{*}, P_{i}, \sigma_{c o l}, n(D)$ are the threshold diameter of a body that can catastrophically break up the body of diameter $D$, the intrinsic collisional probability in units of $\mathrm{km}^{-2} \mathrm{yr}^{-1}\left(P_{i}\right.$ is defined as the probability that a single member of the impacting population will hit the target over a unit of time), the collision cross section, and the number of asteroids per unit diameter, respectively $([17])$.

To compute $P_{i}$ and the average collisional velocity, Bottke et al. ([6]) took a representative sample of MB asteroids (all 682 asteroids with $D>50 \mathrm{~km}$ ) and calculated the collision probabilities and impact velocities between all possible pairs of asteroids, assuming fixed values of semimajor axis, eccentricity, and inclination $(a, e, i)$. Note that so-called Öpik-like codes like that in Bottke et 
al. assume the orbits can be integrated over uniform distributions of longitudes of apsides and nodes; this approximation is considered reasonable because secular precession randomizes the orientations of asteroid orbits over $\approx 10^{4} \mathrm{yr}$ timescales. However, it fails while an object is in a resonance. After all possible orbital intersection positions for each projectile-target pair were evaluated and weighted, it is found that $\mathrm{MB}$ objects striking one another have $P_{i}=$ $2.86 \times 10^{-18} \mathrm{~km}^{2} / \mathrm{yr}$ and a collisional velocity equal to $5.3 \mathrm{~km} / \mathrm{s}$. These values have then been corroborated by different authors and methods. Gravitational focusing is generally neglected because escape velocities from asteroids are $\approx$ $\mathrm{m} / \mathrm{s}$ whereas asteroid impact velocities are of the order of several $\mathrm{km} / \mathrm{s}$. Thus, the collisional cross section is expressed as:

$$
\sigma_{c o l}=\frac{\pi\left(D+D^{*}\right)^{2}}{4} .
$$

The size distribution of asteroids $n(D)$ can undergo some slight changes over time due to the dynamical and collisional evolution of the population, but the current size distribution can still be approximated by analytical forms (e.g., [26]):

$$
n(D) d D=2.7 \times 10^{12} D^{-2.95} d D \text { for } D>100 \mathrm{~m}
$$

Then Eq. 2 can be approximated to ([19]),

$$
\begin{gathered}
\frac{1}{\tau_{c o l}(D)} \sim \frac{P_{i} D^{2}}{4} N(>D) \\
N(>D)=\int_{D_{p}^{*}} n\left(D^{*}\right) \mathrm{d} D^{*}
\end{gathered}
$$

The threshold diameter $D^{*}$ is determined by the impact physics and will be discussed in the next section. A typical value for $D^{*}$ used in the current studies for a $10 \mathrm{~km}$-diameter asteroid is $600-700 \mathrm{~m}$ ([28]). Because the number of asteroids larger than $600-700 \mathrm{~m}$ in diameter calculated by the Eq. 6 is $\sim 5 \times 10^{6}, \tau_{c o l}(D=10 \mathrm{~km}) \sim 3 \mathrm{Gyr}$ in agreement with current estimates of the collision lifetime of $10 \mathrm{~km}$-size asteroid in the MB by the latest collisional evolution models ([52], [9]).

The orbit of small asteroids is changed by the Yarkovsky effect on timescales smaller than their collisional lifetime. The semimajor axis displacement $\delta a$ that a stony body having a diameter of $0.01 \mathrm{~km}$ can undergo is estimated to be about $0.001 \mathrm{AU} / \mathrm{Myr}$, while it is about $2-5 \times 10^{-5} \mathrm{AU} / \mathrm{Myr}$ for $2-4$ $\mathrm{km}$ diameter asteroids ([7]). Thus, the mobility $\delta a$ decreases with the size of the body. These values of $\delta a$ have important dynamical consequences. For instance, $0.01-0.1 \mathrm{AU}$ are typical distances that a $\mathrm{MB}$ meteoroid might have to travel to reach a powerful resonance, and diffusing on such distances would require $10-100 \mathrm{Myr}$ only. Therefore, meteorites do not have to be directly injected into a resonance as a result of their parent body disruption. They can 
rather be created further away from the resonance and then be injected into it as a result of the Yarkovsky effect after a few 10 Myr. Then, although the transport to the Earth once inside the resonance is short (only a few Myr), this scenario explains why the cosmic ray exposure age (CRE, see further) measured for most meteorites is greater than the short transport timescale by resonances (see, e.g., [42]). Similarly, the orbital distribution of members of old families, such as Koronis, can be explained by a combination of the Yarkovsky effect and diffusion in high order resonances, starting from the initial spreading produced by the disruption of the parent body, which is likely narrower than the observed one.

On the course of their dynamical and collisional evolutions, the surface of asteroids is processed in the interplanetary environment. The already mentioned space weathering is one of the material processing that can be remotely detected. The timescale of this process is not well established at present ([54]). The degree of the damage by cosmic ray is analyzed on meteorites. CRE ages of the most common meteorites, i.e. the ordinary chondrites, range from a few Myr to $100 \mathrm{Myr}([37])$. CRE ages give the length of time a body has been exposed in space as a meter-scale object or near the surface of a larger body and therefore can be used to estimate the time between the liberation from the parent body and the arrival on Earth's ground.

\section{Disruption by hypervelocity impact}

\subsection{Initial Shock Pressure and Propagation}

When two small bodies collide with each other with a velocity of the order of $\mathrm{km} / \mathrm{s}$, shock waves are generated and propagate in these bodies. Fig. 2 shows a schematic view of a collision. The shock wave velocity $U_{s}$ is well approximated by a linear relationship with the particle velocity $u_{p}$ for the considered velocity range (see Fig. 3). Its expression is given by:

$$
U_{s k}=C_{B k}+s_{k} u_{p k} \quad(k=p, t)
$$

where the subscripts $p$ and $t$ denote each of the two colliding bodies, i.e. the projectile (conventionally considered as the smaller body involved) and the target. Here $C_{B}$ and $s$ are the bulk sound velocity and a dimensionless constant of the order of unity, respectively. In a planar impact approximation, the pressure at contact $P$ is:

$$
P_{k}-P_{0 k}=\rho_{0 k} U_{s k} u_{p k} \quad(k=p, t)
$$

where $P_{0}$ is the pressure before the compression, which can be neglected in most cases, and $\rho_{0}$ is the bulk density. This is one of the Hugoniot equations (see e.g. [38]). The pressure is determined under the following two boundary conditions at the surface of contact between the two bodies: (1) the pressure

To be published in: "Small Bodies in Planetary Systems". (Mann, Nakamura, Mukai eds.) 
balance and (2) the equality of particle velocity in both bodies. The first condition expresses as:

$$
P_{t}=P_{p}
$$

and the second condition is:

$$
u_{p t}=V_{i}-u_{p p}
$$

where $V_{i}$ is the impact velocity.

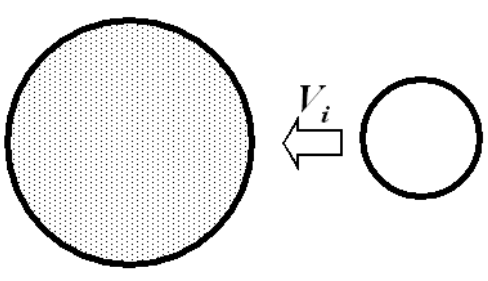

the two bodies are made of the same material, then

$$
u_{p t}=u_{p p}=\frac{V_{i}}{2}
$$

and

$$
P_{p}=P_{t}=\rho_{0}\left(C_{B}+s \frac{V_{i}}{2}\right) \frac{V_{i}}{2} .
$$

The initial shock pressure during the collision is of the order of tens GPa in the MB, where typical collision velocities are of several $\mathrm{km} / \mathrm{s}([6])$. This is about (or more than) 100 times the compressive strength of terrestrial rocks. Since brittle materials are weaker by an order of magnitude in tension than in compression, such mutual collisions naturally lead to the breakup of the small rocky bodies involved in the collision. The level of initial pressure is only marginal with respect to the level required for partial rock melting, which is 


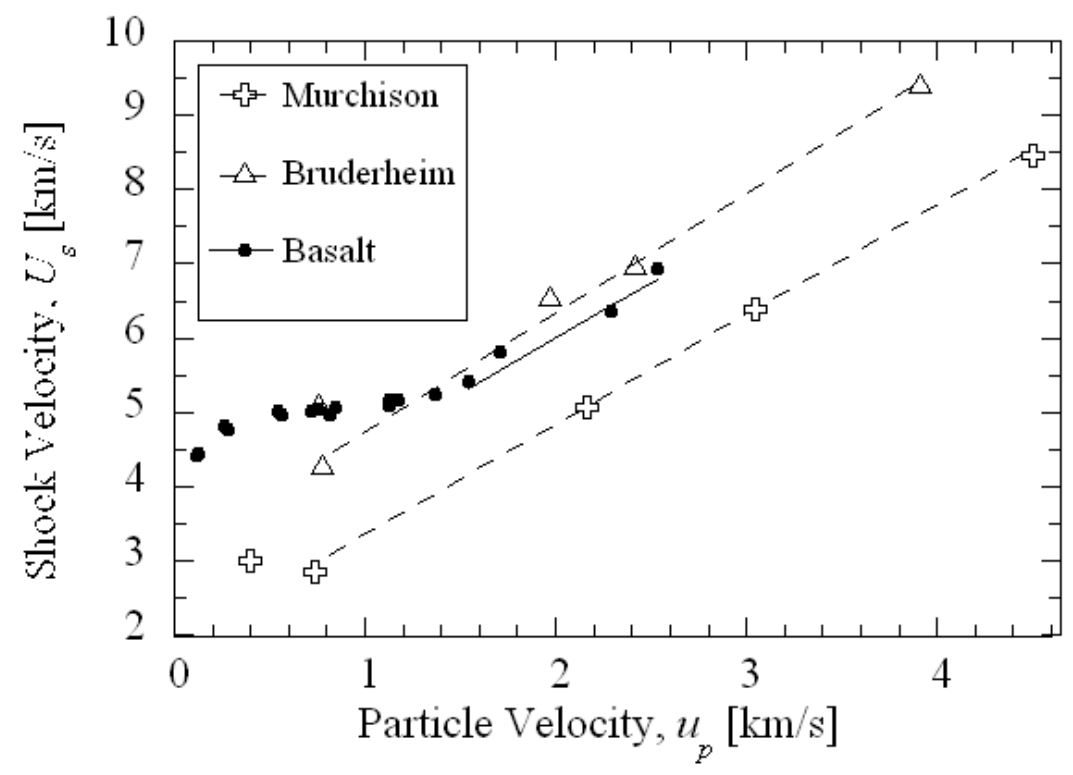

Fig. 3. Relationship between shock and particle velocities for two meteorites, namely Murchison (carbonaceous chondrite, CM2) and Bruderheim (ordinary chondrite, L6) ([2]), and Kinosaki basalt ([49]). The values of the bulk density $\rho_{0}$ are 2.2, 3.3, 2.7 $\mathrm{g} / \mathrm{cm}^{3}$, respectively; those of the bulk sound velocity $C_{B}$ are $1.87,3.11,3.0 \mathrm{~km} / \mathrm{s}$, respectively and those of the constant $s$ are 1.48, 1.62 and 1.5, respectively.

typically in the range between 50 and $100 \mathrm{GPa}([38])$. However, some shock effects are identified on minerals and meteorites at a pressure level less than $50 \mathrm{GPa}$.

The pressure of the shock wave decays in the body with the propagation distance from the impact point, with a rate depending on the material and on the pressure level ([27]). The pressure rapidly decays in a strong shock regime where the particle velocity is much larger than the sound velocity, i.e., $u_{p}>>C_{B}$. Fig. 4 shows a typical decay curve of non-porous rocks in the intermediate regime, where $u_{p} \sim C_{B}$, and illustrates that the initial shock pressure decreases by three orders of magnitude when the wave travels a distance of a few tenths of the projectile's radius.

\subsection{Hyper-Velocity Impact Experiment Conditions}

In a typical set-up, a two-stage light-gas gun is used to accelerate a mm- or $\mathrm{cm}$-size projectile to the velocities involved in asteroidal collisions (several $\mathrm{km} / \mathrm{s}$ ). Powders are fired in the first stage and the combustion gas gives pressure to a plastic piston which in turn compresses a light gas in the second stage. The projectile is accelerated by the light gas, i.e. $\mathrm{H}$ or $\mathrm{He}$, and the ve- 


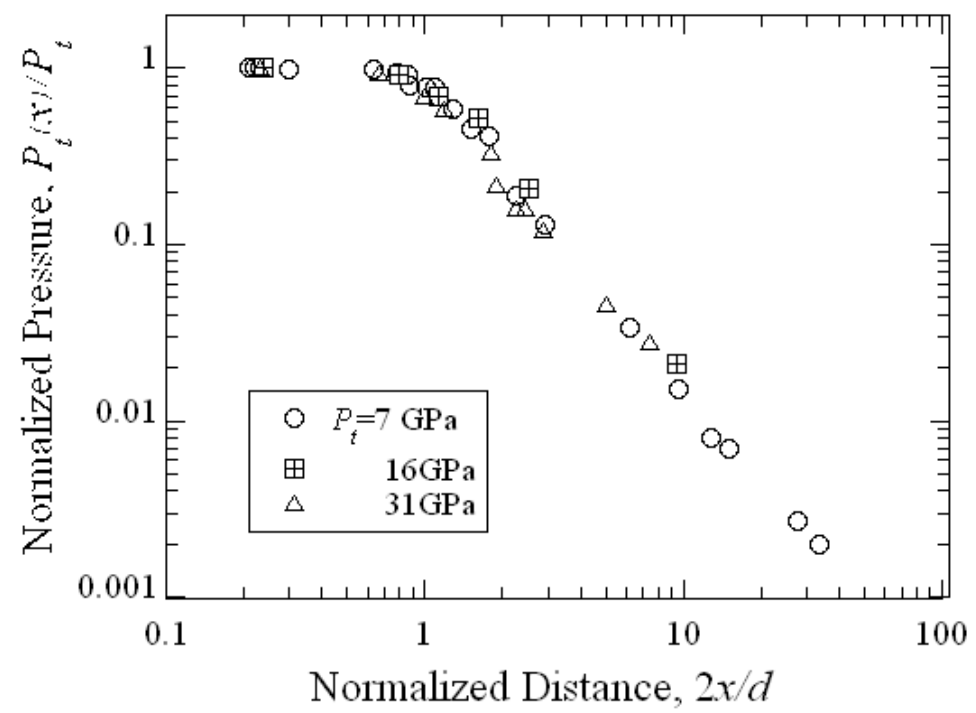

Fig. 4. Experimentally determined shock pressure decay in Kinosaki basalt ([50]). The pressure $P_{t}(x)$ at the propagation distance $x$ is normalized by the initial peak pressure $P_{t}$. The radius $d / 2$ of the projectile was $4.5,4.5$ and $14 \mathrm{~mm}$ for experiments with initial $P_{t}=31,16$ and $7 \mathrm{GPa}$, respectively. The projectile's shape was a disk (with thickness about one ninth of the diameter) for the 31 and $7 \mathrm{GPa}$ experiments and a cylinder (with height equal to the diameter) in the $16 \mathrm{GPa}$ experiment.

locity is limited by the sound velocity of the light gas. Projectiles are usually composed of plastics or metals. To avoid that the projectile breaks in the gun muzzle, a sabot is used to accelerate the projectile. As for the targets, a wide variety of materials have been used, including rocks, ices, and even sands in a thin paper bag ([67]).

Material properties of the targets, which have important influence on the collisional and cratering processes, are the bulk density, the yield strength, the compressive, shear, and tensile strengths ([3], see also Michel's chapter in this issue), the porosity, the longitudinal and transversal wave velocities, and the Hugoniot parameters (e.g., $C_{B}$ and $s$ in Eq. 7). The measurements of these material properties have been performed for terrestrial rocks, although some of them require sophisticated instrumentations. Other material parameters used in numerical simulations of impact disruption are the Weibull parameters, which represent the distribution of incipient flaws in the material. These parameters have recently been measured for one of the materials used in previous impact disruption experiments (see Sec. 3.3). Among all these material parameters, only the bulk density and inferred porosity have been determined for some asteroids. Other material properties have not been measured directly 
for asteroids and comets, so all our understanding of the collisional process relies on terrestrial rocks and meteorites.

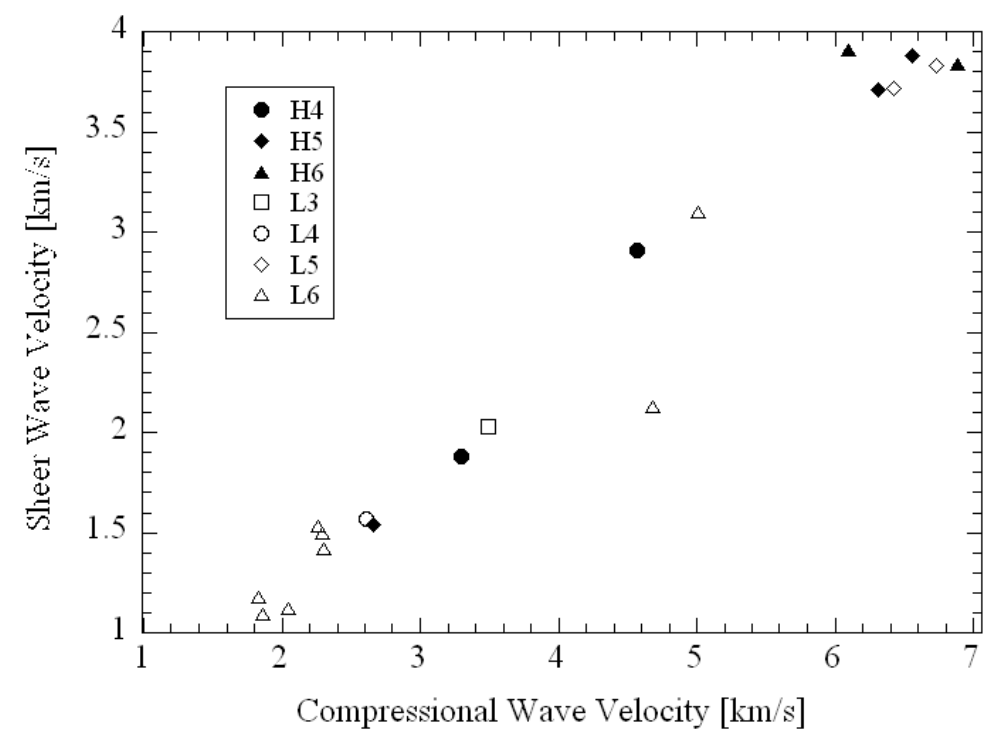

Fig. 5. Elastic wave velocities in L-type and H-type ordinary chondrites ([69]) and terrestrial rocks used in previous analogue impact experiments ([61]).

Meteorites are the only materials available in laboratory which are more or less directly connected to some asteroids. However, meteorites have only been used in a few impact experiments, due to the small volumes of sample available. Therefore, terrestrial rocks and synthesized materials are more commonly used. Fig. 5 shows elastic wave velocities in ordinary chondrites ([69]) and in materials used in previous analogue impact experiments ([61]). Figs. 3 and 5 indicate that the terrestrial rocks used in previous analogue experiments have some of their mechanical properties which are similar mechanical properties to those of ordinary chondrites. However, there are three distinct problems that prevent to make a direct link between the material constituing meteorites and asteroid material. First, we do not have samples for of all the asteroid taxonomic classes. In particular, our collection is likely to be biased toward material coming from the inner part of the asteroid belt. Second, our collection has probably suffered greatly from a selection effect against weaker materials, as only the strongest material can survive the dynamical pressure undergone during atmospheric entry and transit. For example, analyses of the trajectory of a meteorite's fall have suggested that meter-class, stony, near-Earth asteroids (NEAs) have tensile strengths more than an order of magnitude lower than those measured for ordinary chondrites ([11]). Third, 
there is a great difference between asteroids and meteorites in size scale. It is known that the strength of brittle materials decreases with size, because larger rocks have statistically a higher probability to contain a weaker part (larger incipient flaws) in their volume than smaller rocks. This will be discussed in the next section. Moreover, asteroids are found to have a smaller bulk density than taxonomically corresponding meteorites, indicating higher internal porosity in those bodies than in meteorites.

Therefore, although meteorites are rich in information (in particular concerning the chronology of our Solar System), their physical properties may not be representative of those of the material that evolves in space. Thus, our understanding of the material properties of asteroids is still very limited. Similarly, our understanding of material behavior under impacts is limited to terrestrial rocks, and we can just hope that our findings can still provide us information that are relevant to asteroid disruption events.

\subsection{Distribution of incipient flaws in a rock: the Weibull parameters}

Natural materials intrinsically have non-uniform physical properties. This non-uniformity often becomes the major source of scattering in the outcome of collisional disruptions of rocks in laboratory experiments under same initial conditions. For example, everything else being equal in experimental conditions, targets cut out from a same large block of rock break differently one another, due to the one by one different strength of these targets. The statistical behavior of the material strength is usually expressed by a Weibull distribution:

$$
n(\sigma)=K\left(\frac{\sigma}{\sigma_{N}}\right)^{m}
$$

where $n(\sigma)$ is the density number of flaws in a rock that activate at a stress not greater than $\sigma([66]), K$ is constant, and $m$ and $\sigma_{N}$ are the constant Weibull parameters (note that in the litterature, the Weibull parameters are indicated to be $m$ and $k$, where $k$ is related to $K$ and $\sigma_{N}$ ). The probability of failure $P_{\text {probability }}$ of a specimen of a given volume at the stress $\sigma$ is represented by:

$$
P_{\text {probability }}(\sigma)=1-\exp \left[-\left(\frac{\sigma}{\sigma_{N}}\right)^{m}\right]
$$

where $m$ and $\sigma_{N}$ are called shape and scale, respectively. The value of $m$ gives an indication of the degree of homogeneity within the material. Higher values of $m$ indicate that flaws are more evenly distributed throughout the material, and consequently, the strength is nearly independent of the volume of the specimen. Lower values of $m$ indicate that flaws are fewer and less evenly distributed, causing greater scatter in strength. The characteristic stress $\sigma_{N}$ 
is the stress at which $63.2 \%$ of similar units subjected to failure will have failed.

It is only recently that the Weibull parameters of the target material used in impact experiments have been experimentally determined ([47]). Fig. 6 shows the X-ray CT-scan image of a basalt target used in previous impact disruption experiments and the measured result of the probability of failure under static loading. The value of the shape parameter $m$ is in the range 15-17 for this material and is higher than the value used in numerical simulations of the impact disruption of this material (using about $9-9.5$ ).
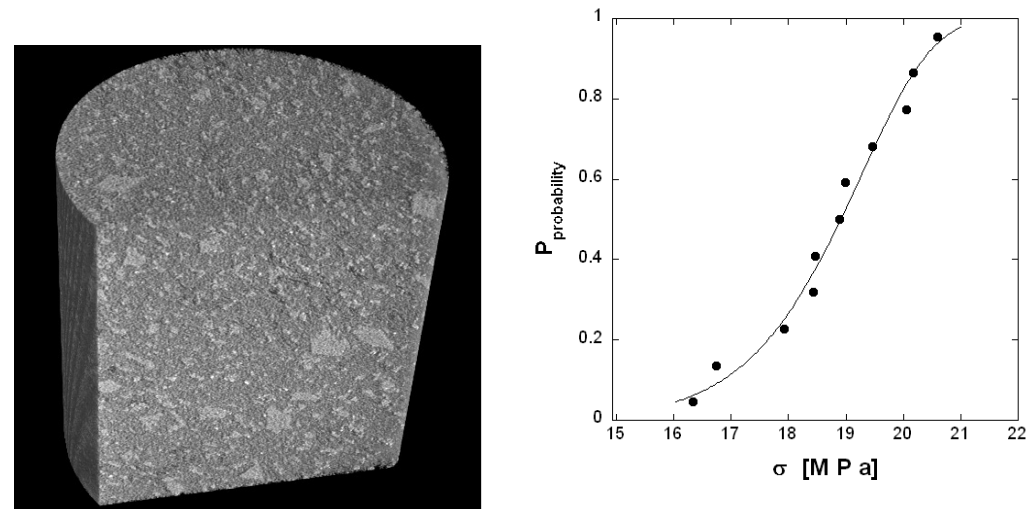

Fig. 6. Left: X-ray CT-scan image (diameter is $4.2 \mathrm{~mm}$, taken by XMS-BS9, Microscopic Scan Co.) of a basalt target used in some impact experiments with in-depth data ([44], [45]). Right: Probability of failure $P_{p}$ robability in a diametral compression test of a basalt specimen of diameter $10 \mathrm{~mm}$ and thickness $5 \mathrm{~mm}$. ([47])

The dependency of material strength on target's size is directly related to the Weibull distribution of incipient flaws. Indeed, the minimum stress $\sigma_{\min }$ at which a flaw in a target of volume $V$ activates is derived from $n\left(\sigma_{\min }\right)=$ $1 / V=K\left(\sigma_{\min } / \sigma_{N}\right)^{m}$. Thus, we have:

$$
\sigma_{\min }=\sigma_{N}(K V)^{-1 / m} .
$$

The threshold for failure $\sigma_{\min }$ thus goes with the $-3 / m$ power of the target's size, and therefore, larger targets start to break at lower stresses than smaller ones. Hence, for $m=6$, the strength is poportional to $r^{-1 / 2}$, where $r$ is the body's radius, and such a decrease of strength with body's size is often used in collisional evolution models. 


\subsection{Outcome of collisional disruption}

\section{Largest fragment}

One of the most fundamental result of an impact disruption is the degree of fragmentation as a function of the impact initial conditions. The mass fraction of the largest fragment to the original target is commonly used as the indicator of the degree of fragmentation. When the stress level of the wave in the target at the antipodal point from the impact point is larger than the tensile strength of the target, the antipodal surface is removed (broken off) due to tensile failure and the largest fragment corresponds to an internal part of the target. It is usually called in this case a core fragment. Fine fragments are rather generated in the vicinity of the impact point. Such tensile failure at the target surface is called spallation and is also seen in laboratory cratering impacts on brittle materials. Fig. 7 shows an example of a core fragment. On the other hand, when the stress level of the wave in the target is low due to either poor transmissivity of the shock wave by the target material or low initial pressure, spallation does not occur and cone-shaped largest fragments with their tops heading at the impact point are created.

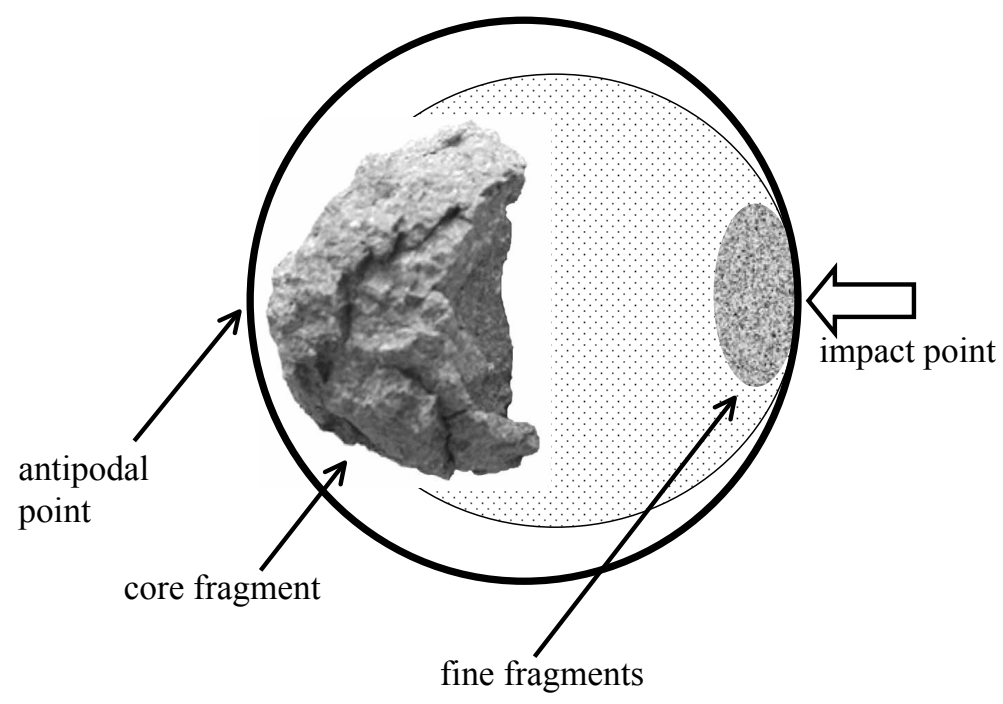

Fig. 7. A core fragment from a $6 \mathrm{~cm}$ diameter basalt disruption experiment ([45]).

The most direct indication of the impact condition is the energy density, also called specific impact energy $Q$, defined as the kinetic energy of the pro- 
jectile (the smaller of the two colliding bodies) divided by the mass of the target in the frame where the target is at rest. Because the initial pressure and the following pressure decay are different depending on the impact velocity or the impacting materials (e.g., a iron-rock collision involves much higher initial pressure than a rock-rock collision), they cannot be used as an intrinsic indicator for impact events with different impact velocities or involving very different materials from solid rocks. Conversely, the energy density is commonly used as a reference not only in laboratory experiments but also in numerical simulations of the collisional disruption and evolution of asteroids. The threshold value for catastrophic disruption, $Q^{*}$, is defined as the impact energy required to produce a largest remaining piece whose mass corresponds to $50 \%$ of the mass of the original body ([22], [28]). Then assuming that both bodies have the same bulk density, the projectile diameter $D^{*}$ needed for catastrophically disrupting an object of diameter $D$ with an impact velocity $V_{i}$ is

$$
D^{*}=\left(\frac{2 Q^{*}}{V_{i}^{2}}\right)^{(1 / 3)} D
$$

Generally, it is determined for a fixed impact velocity, and its trend is analysed as a function of the target's size by adjusting the projectile's mass. Usually, impact velocities consistent with the ones in the MB (about $5 \mathrm{~km} / \mathrm{s}$ ) are considered, but then, one should note that the curves of $Q^{*}$ as a function of diameter published for this velocity, in principle, cannot be used for collisions made at other velocities.

The specific impact energy to shatter an object, $Q_{S}^{*}$, is defined as the threshold value at which the largest remaining intact piece immediately following the fragmentation contains $50 \%$ of the mass of the original body. We refer to it as the shattering energy. At large body sizes (above a few hundreds meters), the shattered pieces may reaccumulate due to their mutual gravitational attractions, depending on their velocity relative to their mutual escape velocity. Therefore, at those large sizes, in the so-called gravity regime, a higher impact energy threshold $Q_{D}^{*}$ is defined and corresponds to the specific energy such that the largest fragment (which may be produced by reaccumulation of smaller ones) contains $50 \%$ of the mass of the original body. This is called the threshold energy for disruption. Note that in the strength regime where gravity is negligible, $Q_{S}^{*}=Q_{D}^{*}$. A typical value of $Q_{S}^{*}$ for a cm-scale rocky target is $\approx 10^{7} \mathrm{erg} / \mathrm{g}$ at impact velocities consistent with $\mathrm{MB}$ values. The value of $Q_{S}^{*}$ is extrapolated at larger target's sizes from the experimental values at $\mathrm{cm}$-size by numerical simulations or using some scaling laws. However, due to our poor understanding of the process of fragmentation, depending on the assumptions and models used, the extrapolated value of $Q_{S}^{*}$ can vary by several orders of magnitude at a given size ([28]). Although the gravity regime should be better understood, as Newton's law of gravity is well-known compared to the concept of solid strength, the body's size at which the transition occurs between the strength and gravity regime (where

To be published in: "Small Bodies in Planetary Systems". (Mann, Nakamura, Mukai eds.) 
$Q_{S}^{*}$ starts to differ with $Q_{D}^{*}$ ) is still a subject of debates and is somewhere in the range between a few hundreds meters to a few kilometers according to various studies. Small body populations are composed of bodies with a wide range of material properties, and several evidence point toward the presence of bodies with a high degree of porosity (e.g., [10], [23]). As a consequence, determining the outcome of the disruption of a porous body is now considered to be extremely important for studying the origin and collisional evolution of small body populations, and for determining efficient mitigation strategies against a threatening NEO. Generally, the impact energy threshold for disruption depends on material strength and gravity when the body is large enough ([28]); however, porosity plays a complicated role. The static compressive strength $(S)$ of porous material is usually lower than that of dense material, as indicated by an empirical formula known for ceramics ([57]), given by $S=c_{1} e^{-c_{2}(1-\phi)}$, where $c_{1}$ and $c_{2}$ are constant, and $\phi$ denotes the filling factor, $(1-\phi)$ being the porosity. On one hand, with increasing porosity, the target body has a weaker tensile strength. On the other hand, the increasing volume of void space decreases the transmission efficiency of stress waves in the target body, so that porous bodies appear stronger against impact.

The impact response of a small body, and consequently, the mass of the largest remnant from its disruption are thus highly sensitive to material properties and especially to the degree of porosity. Therefore, it is crucial to have a better knowledge of the collisional process as a function of the internal structure of the bodies, and to determine those properties for the real objects belonging to the different populations of small bodies. This last part will require the development of in-situ space missions toward different bodies, as from the ground, only a limited knowledge can be inferred from the light emitted from their surface.

\section{Fragment size, shape, velocity and spin distributions in small-scale laboratory experiments}

In laboratory experiments, the masses of the largest fragments are measured. The masses (sizes) of largest fragments are highly dependent on the impact conditions such as the energy density and the geometry of the collision characterized by the target's shape and the impact angle of the projectile. The size distribution of smaller fragments is derived from either the masses of individual fragments or from the total mass of fragments in each size range binned by sieves with different opening sizes ([61]). The size distribution of smaller fragments whose sizes are typically equal to or smaller than $\sim 1 \mathrm{~mm}$ in usual laboratory cm-scale disruption experiments, is not highly sensitive to the impact conditions. The differential size distribution of fragments from a rocky body can often be well fitted with a power index between -3 and -3.5 . Interestingly, a power-law exponent of -3.5 is also used for the size distribution of interstellar dust grains (MRN-distribution [35]). The size distribution of even smaller fragments (fine fragments) with sizes less than $10 \mu \mathrm{m}$ is investigated 
at specific directions from the impact point using witness plates ([46]). The size distribution of fine fragments from basalt targets shows similar power-law index to that of $\sim 10 \mu \mathrm{m}-1 \mathrm{~mm}$ fragments. The ones for chondrites also show a similar tendency $([20])$.

The shape of fragments is investigated using high-speed images taken during impact experiments or is directly measured using a slide caliper. The shape is charaterized by the axial ratios, $\mathrm{B} / \mathrm{A}$ and $\mathrm{C} / \mathrm{A}$, in an ellipsoid approximation (with $\mathrm{A}>\mathrm{B}>\mathrm{C}$ ). One method starts by measuring the largest dimension of the fragment, $\mathrm{A}$, and the other method starts by measuring the smallest dimension of the fragment, C. These two main methods of measurement of the axes can lead to different results. Fragments from catastrophic disruptions rarely have $\mathrm{B} / \mathrm{A}$ or $\mathrm{C} / \mathrm{A}$ ratios below 0.3 and 0.2 , respectively. The mean values cluster around 0.7 and 0.5 over widely different experimental conditions ([22]). In cratering events, larger fragments are presumably spall fragments and have plate-like shapes. It has been reported that most of the spall fragments from craters excavated in Gabbro had B/A values greater than 0.6 and $\mathrm{C} / \mathrm{A}$ values less than 0.25 ([55]).

The surface roughness of fragments is investigated by fractal analysis. The fractal dimension is not dependent on the degree of fragmentation and the value determined by a divider method is $\sim 2.1-2.4$ (see [32]).

In general, fragment velocities and rotational frequencies are the highest near the impact point and decrease with increasing distance from this site. Surface fragments tend to have higher velocities than fragments from the interior of the target. The upper bounds of the fragment velocity and rotational frequency distributions usually decrease with increasing fragment's size ([28]).

\section{Impact Process on Asteorid 25413 Itokawa}

In 1991, a MB S-class asteroid 951 Gaspra was visited during a fly-by of the NASA Galileo spacecraft ([4]). Gaspra has an irregular shape of $19 \times 12 \times 11$

$\mathrm{km}$ and has a great number of small craters and grooves on its surface as well as color variations suggesting some space weathering effect. In 1993, the Galileo spacecraft made a fly-by to another MB S-class asteroid called 243 Ida. ([5]). Ida is a body of $58 \mathrm{~km}$ long. It belongs to the Koronis family and was found to have a $1.4 \mathrm{~km}$ diameter moon, called 243(1) Dactyl. Owing to the discovery of this tiny satellite, the bulk density of Ida was determined to be $2.6 \mathrm{~g} / \mathrm{cm}^{3}$. Next, a MB C-class asteroid 253 Mathilde was visited during a fly-by of the NASA NEAR spacecraft ([64]). Mathilde is a $66 \times 48 \times 46 \mathrm{~km}$ body with a very slow rotational period of 17.4 days. The bulk density of the body was found to be $1.3 \mathrm{~g} / \mathrm{cm}^{3}$. This small value led to the conclusion that the asteroid contains $40 \%$ or more macroporosity. There are at least five craters larger than $5 \mathrm{~km}$ in size on Mathilde and this was also interpreted as a piece of evidence that Mathilde has a high fraction of vacuum space inside, which allowed the whole body to survive the impacts that created those craters. The

To be published in: "Small Bodies in Planetary Systems". (Mann, Nakamura, Mukai eds.) 
high porosity effectively attenuates the shock waves generated by such impacts and helps to maintain the whole body intact. Otherwise, it was believed that the impact energy needed to form such large craters should have disrupted the body if there was no dissipation mechanism of this energy. Although Mathilde has such a high porosity, it is not clear if Mathilde is a re-accumulated body from a major impact event. Mathilde may be as likely a primitive porous body. In February 2000, the NEAR spacecraft was inserted into orbit around 433 Eros, the second largest NEO, and was renamed the NEAR-Shoemaker spacescraft ([65]). Eros is a S-class NEO whose size is $33 \times 13 \times 13 \mathrm{~km}$. Its bulk density is $2.67 \mathrm{~g} / \mathrm{cm}^{3}$, which is close to the value estimated for Ida. During the $\sim 1$-year mission in orbit around Eros, more than a hundred of thousands images were taken. The presence of global ridges and grooves and higher order gravitational data all indicate that Eros has at least partially a cohesive, homogeneous interior in spite of $\sim 20 \%$ of macroporosity. This percentage of porosity is inferred from spectral data which suggest that Eros has a similar composition to ordinary chondrites. Thus, the total porosity can be estimated from the ratio of the bulk density of Eros $\left(2.67 \mathrm{~g} / \mathrm{cm}^{3}\right)$ and the grain density of the component minerals $\left(3.75 \mathrm{~g} / \mathrm{cm}^{3}\right.$, when assuming L-chondrites composition) to be $(1-2.67 / 3.75) \times 100=28.8 \%$. Since the microporosity of L-chondrites is on average $10.8 \%$, assuming a same level of microporosity in Eros leads to a macroporosity of $18 \%$ within this asteroid ([10]). Therefore, S-class bodies are usually not interpreted as being extremely porous and their porosity is rather considered as macroporosity. Conversely, the porosity indicated for dark-type (e.g. C-class) bodies is considered as being composed of microporosity at higher levels in addition to large voids and fractures. The material constituing these bodies is thus believed to be porous at micro-scale, which makes them behave differently than non-porous (or macro-porous) bodies during impact phenomena.

In 2005, the JAXA Hayabusa spacecraft performed a sample return mission to the S-class NEO 25143 Itokawa, whose length is about 500 meters. Although the return of the sample is not guaranteed yet, the mission is already considered as a great success in the scientific community, given the wealth of new information and surprizes that it provided by the images and measurements made during the visit of the spacescraft. Itokawa is the smallest asteroid that has ever been visited by an artificial satellite. The largest boulder (Yoshinodai) lying on its surface is one-tenth the size of the whole asteroid. Yoshinodai and other ten meter-size boulders are too large to be generated from any impact crater candidates on Itokawa. The bulk density of Itokawa is $1.9 \mathrm{~g} / \mathrm{cm}^{3}([1])$ and is far below the values found for other S-class asteroids. These facts collectively strongly suggest that Itokawa is a gravitationally re-accumulated asteroid from a major impact disruption of a parent asteroid ([23]). Therefore, images of the boulders on Itokawa's surface with pixel resolution of $\sim 6 \mathrm{~mm}$ to $\sim 70 \mathrm{~cm}$ taken by the Asteroid Multi-band Imaging CAmera (AMICA) provide us with the actual outcome of the collisional disruption event of the parent body of a subkilometer-size asteroid. 


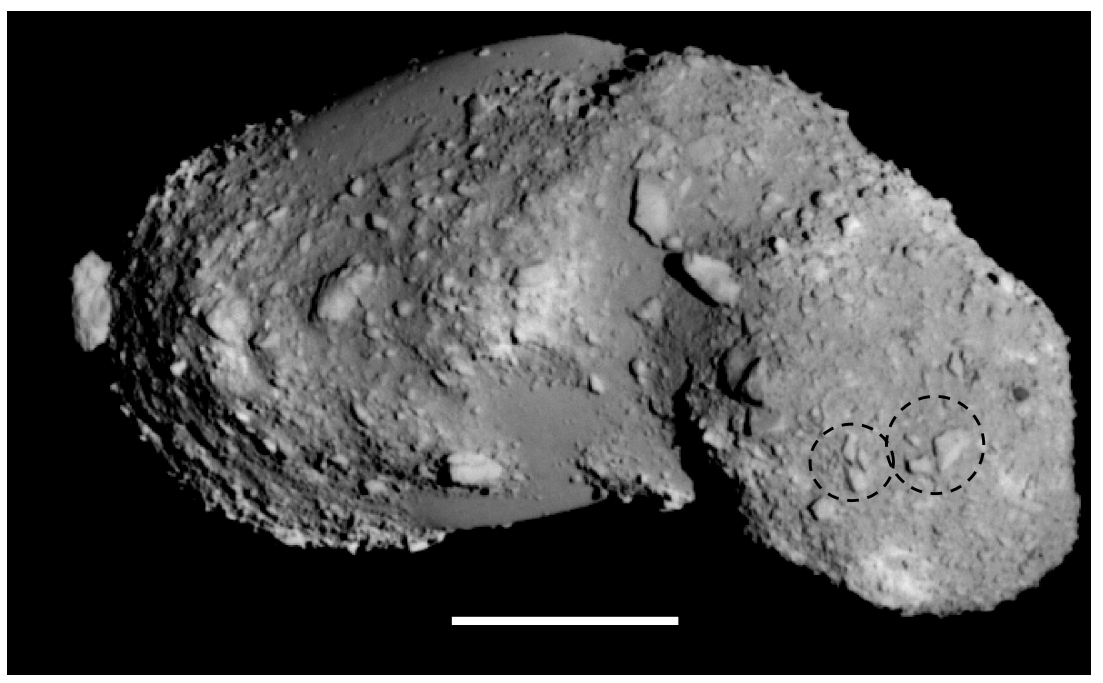

Fig. 8. An overview of the asteroid 25143 Itokawa ((c) ISAS/JAXA). Scale horizontal bar $=100 \mathrm{~m}$. The boulder at the left end is the largest boulder (Yoshinodai). Boulders in circles appear as if they were originated from a single larger boulder or two large boulders which underwent cratering impact on Itokawa's surface ([47]).

The boulders on Itokawa exhibit a wide spectrum of angularities and irregularities. There are thin, flat-looking boulders, angular and conical boulders, and irregularly shaped (wavy-shaped) boulders. A similar variety is also found for fragments in laboratory. Fig. 9 shows a comparison of boulders on Itokawa and laboratory fragments from centimeter-size targets collected after impact experiments. Cracks and fractures are observed both on boulders and experimental fragments.

Although there is a difference of many orders of magnitude in the scale and complexity of the physical processes, as well as in the environment (gravity), the similarities in shapes and structures of the boulders and the laboratory fragments establish a bridge between disruption in laboratory of solid bodies (governed by the growth and coalescence of microscopic flaws) and the natural collisional disruption process at larger scales in space. These similarities suggest a universal character of at least some parts of the process throughout these scales.

In principle, the impact process related to the boulders on Itokawa's surface is expected to be erosive because of the tiny gravitational attraction of the body. The fraction of ejecta from a cratering process that have velocities less than the escape velocity is dependent upon the strength of the surface ([29]) if the crater is excavated on a cohesive surface. In the case of Itokawa, the internal structure cannot be inferred with great details, but it is likely that the thickness of the boulder layer does not exceed a few tens of meters. When 

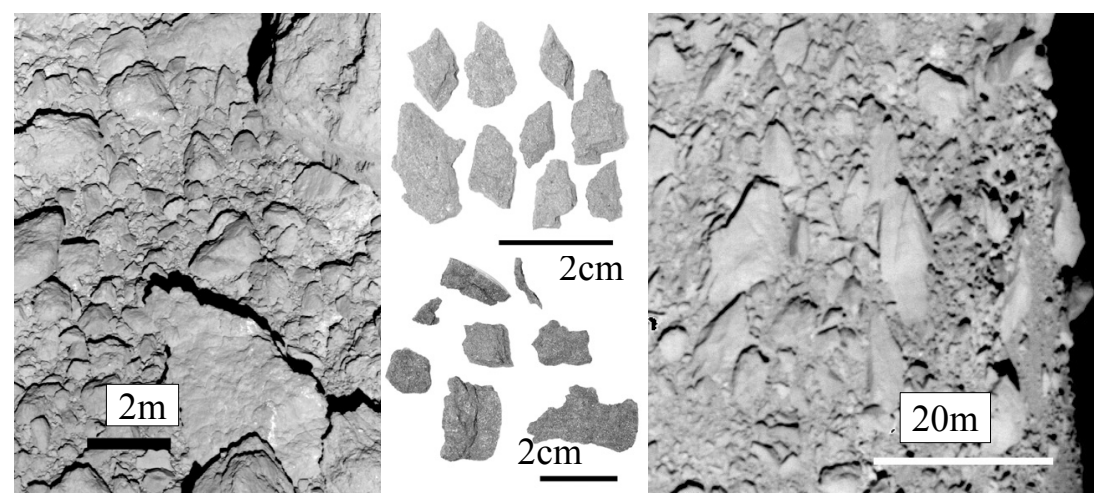

Fig. 9. Irregularly shaped fragments from laboratory impact experiments and boulders on the surface of Itokawa ((c) ISAS/JAXA, [48])

the impactor is large enough to penetrate into the boulder layer and the bedrock, the fraction of the falling-back ejecta is governed by the strength of the bedrock. Similarly, when the impactor is smaller than the size of individual boulders, the fraction of the ejecta from a crater excavated on a boulder itself that can fall back onto the surface is also controlled by the strength of the boulder material. Since the escape velocity from the surface of Itokawa is only $10-20 \mathrm{~cm} / \mathrm{s}$, the fraction of the ejecta that can fall back on the surface is expected to be very small ([40]), unless there is a great dissipation of energy during cratering events. Therefore, most of the boulders on the surface should rather come from the collisional disruption event that formed Itokawa and may also correspond to original boulders of the parent body of Itokawa. The slope of the cumulative size distribution of boulders on Itokawa's surface is, however, close to -3 ([40]) which is steeper than the size distributions of fine fragments in laboratory disruptions. One possible explanation of this discrepancy is that some boulders may not be genuine in the sense that some of them may have originated from a single larger boulder which was broken up during its fall back on the surface, or later by a cratering event. In fact, boulder pairs or groups (or families) have been indentified on Itokawa's surface as shown in Fig. 9. These families of boulders might well be the remnants of the impact disruptions of larger boulders on the surface ([48]). The other explanation is that at least some of the boulders on the surface of Itokawa original boulders belonging to the parent body of Itokawa.

\section{Summary and perspectives}

More than two hundred years have passed since the first asteroid 1 Ceres was found in 1801 in Palermo by G. Piazzi ([21]), and our knowledge on 
asteroids has been changed qualitatively and continuously during the last decades. Asteroids appear more active and complicated than ever thought. Many asteroid-satellite and binary systems are being discovered and provide information on asteroid's masses and bulk densities. The bulk density, which is lower than typical bulk densities of small rocks, tells us that asteroid interiors contain generally substantial void spaces. Moreover, the dynamical evolution of asteroids appears more complex as it is controlled not only by Kepler's laws, but also by interaction with photons, e.g. the Yarkovsky thermal effect. The interaction with photons also affects the spin state of these bodies when their shape is not spherical, which is the case of almost all of them, and when they are small enough, and now there are a few observational evidence showing that this so-called YORP effect has changed some real asteroid spin periods ([34], [31]). Another observation shows the evidence of volatile activity on the surface of bodies which reside in the main belt ([30]).

Although our understanding of the collisional evolution of asteroids suggests that most of the ones whose size is at most a few tens of kilometers have experienced major catastrophic disruption events and have fractured or rubble-pile internal structures (in agreement with measured bulk densities), only one sub-km asteroid, the smallest ever explored 25413 Itokawa, have shown direct evidence that it is a gravitationally re-accumulated body. However, the detailed internal structure and birth scenario of Itokawa has not been revealed yet. Space missions aiming at making a sample return and/or investigating deep into the interior using techniques such as radar tomography are thus strongly required.

Studies of the collisional disruption of asteroids are performed by three different approaches: laboratory experiments, numerical simulations, and theoretical scaling considerations. Laboratory disruption experiments of rocky materials have provided a first order understanding of the collisional disruption process of solid bodies. Now our study of collisinal disruption of such bodies is to be extended to porous ones. Such extension, and its extrapolation at larger sizes by numerical models will lead to a greater understanding of the collisional response and evolution of the small bodies, in particular those which evolve in the outer part of the main belt and further, e.g. Trojans asteroids, Kuiper belt objects and comets. It will then also be possible to address the fundamental problem of collisional accretion which, during the early history of our Solar System, led to the formation of our planets.

\section{References}

1. S. Abe, T. Mukai, N. Hirata, O.S. Barnouin-Jha, A.F. Cheng, H. Demura, R.W. Gaskell, T. Hashimoto, K. Hiraoka, T. Honda, T. Kubota, M. Matsuoka, T. Mizuno, R. Nakamura, D.J. Scheeres, and M. Yoshikawa: Mass and Local Topography Measurements of Itokawa by Hayabusa, Science 312, 1344 (2006)

To be published in: "Small Bodies in Planetary Systems". (Mann, Nakamura, Mukai eds.) 
2. W.W. Anderson and T.J. Ahrens: Shock Wave Equations of State of Chondritic Meteorite, In: Shock Compression of Condensed Matter - 1997, ed. by S. C. Schmidt, et al. (AIP Press, Woodbury, NY, 1998) pp. 115-118.

3. W. Benz and E. Asphaug: Impact simulations with fracture. I-Method and tests, Icarus 107, 98 (1994)

4. J. Veverka, M.J.S. Belton, K.P. Klaasen, and C.R. Chapman: Galileo's Encounter with 951 Gaspra: Overview, Icarus 107, 2 (1994)

5. M.J.S. Belton, C.R. Chapman, K.P. Klaasen, A.P. Harch, P.C. Thomas, J. Veverka, A.S. McEwen, and R.T. Pappalardo: Galileo's Encounter with 243 Ida: an Overview of the Imaging Experiment, Icarus 120, 1 (1996)

6. W.F. Bottke Jr., M.C. Nolan, R. Greenberg, and R.A. Kolvoord: Velocity distributions among colliding asteroids, Icarus 107, 255 (1994)

7. W.F. Bottke Jr., D. Vokrouhlicky, D.R. Rubincam, and M. Broz: The Effect of Yarkovsky Thermal Forces on the Dynamical Evolution of Asteroids and Meteoroids, In: Asteroids III, ed. by W. F. Bottke Jr, A. Cellino, P. Paolicchi and R. P. Binzel (Univ. Arizona Press, Tucson, 2002) pp. 395-408.

8. W.F. Bottke Jr., A. Cellino, P. Paolicchi, and R.P. Binzel, eds. Asteroids III, Univ. Arizona Press, Tucson (2002)

9. W.F. Bottke, Jr., D.D. Durda, D. Nesvornỳ, R. Jedicke, A. Morbidelli, D. Vokrouhlickỳ, and H.F. Levison.: Linking the collisional history of the main asteroid belt to its dynamical excitation and depletion, Icarus 179, 63 (2005)

10. D.T. Britt, D. Yeomans, K. Housen, and G. Consolmagno: Asteroid Density, Porosity, and Structure, In: Asteroids III, ed. by W. F. Bottke Jr, A. Cellino, P. Paolicchi and R. P. Binzel (Univ. Arizona Press, Tucson, 2002) pp. 485-500.

11. P. Brown, D. Pack, W.N. Edwards, D.O. Revelle, B.B. Yoo, R.E. Spalding, and E. Tagliaferri: The orbit, atmospheric dynamics, and initial mass of the Park Forest meteorite, Meteorit. Planet. Sci. 39, 1781 (2004)

12. S.J. Bus, F. Vilas, and M.A. Barucci: Visible-Wavelength Spectroscopy of Asteroids, In: Asteroids III, ed. by W. F. Bottke Jr, A. Cellino, P. Paolicchi and R. P. Binzel (Univ. Arizona Press, Tucson, 2002) pp. 169-182.

13. C.R. Chapman, D. Morrison, and B. Zellner: Surface properties of asteroids: A synthesis of polarimetry, radiometry, and spectrophotometry, Icarus 25, 104 (1975)

14. C.R. Chapman, J. Veverka, P.C. Thomas, K. Klaasen, M.J.S. Belton, A. Harch, A. McEwen, T.V. Johnson, P. Helfenstein, M.E. Davies, W.J., Merline, and T. Denk: Discovery and Physical Properties of Dactyl a Satellite of Asteroid 243 Ida, Nature 374, 783 (1995)

15. B.E. Clark, B. Hapke, C. Pieters, and D. Britt: Asteroid Space Weathering and Regolith Evolution, In: Asteroids III, eds. by W. F. Bottke Jr, A. Cellino, P. Paolicchi, and R. P. Binzel (Univ. of Arizona Press, Tucson, 2002) pp. 585-599.

16. D.R. Davis, S.J. Weidenschilling, P. Farinella, P. Paolicchi, and R.P. Binzel: Asteroid collisional history: Effects on sizes and spins, In: Asteroids II, eds. by R.P. Binzel, T. Gehrels, and M. Matthews (Univ. of Arizona Press, Tucson, 1989) pp. 805-826.

17. P. Farinella, D.R. Davis, A. Cellino, and V. Zapplà: The collision lifetime of asteroid 951 Gaspra, Astron. Astrophys. 257, 329 (1992)

18. P. Farinella and D.R. Davis: Short-Period Comets: Primordial Bodies or Collisional Fragments?, Science 273, 938 (1996)

19. P. Farinella, D. Vokrouhlický, W.K. Hartmann: Meteorite delivery via Yarkovsky orbital drift, Icarus 132, 378 (1998) 
20. G.J. Flynn, D.D. Durda, J.W. Kreft, I. Sitnitsky, M. Strait: Catastrophic Disruption Experiments on the Murchison Hydrous Meteorite, Lunar and Planetary Science XXXVIII, March 2007, Texas, LPI Contribution 1338, p.1744.

21. G. Fodera Serio, A. Manara, and P. Sicoli: Giuseppe Piazzi and the Discovery of Ceres, In: Asteroids III, eds. by W. F. Bottke Jr, A. Cellino, P. Paolicchi, and R. P. Binzel (Univ. of Arizona Press, Tucson, 2002) pp. 17-24.

22. A. Fujiwara, P. Cerroni, D.R. Davis, E.V. Ryan, M. Di Martino, K. Holsapple, and K. Housen: Experiments and scaling laws for catastrophic collisions, In: Asteroids II, eds. by R.P. Binzel, T. Gehrels, and M. Matthews (Univ. of Arizona Press, Tucson, 1989) pp. 240-268.

23. A. Fujiwara, J. Kawaguchi, D.K. Yeomans, M. Abe, T. Mukai, T. Okada, J. Saito, H Yano, M. Yoshikawa, D.J. Scheers, O. Barnouin-Jha, A.F. Cheng, H. Demura, R.W. Gaskell, N. Hirata, H. Ikeda, T. Kominato, H. Miyamoto, A.M. Nakamura, R. Nakamura, S. Sasaki, and K. Uesugi: The Rubble-Pile Asteroid Itokawa as Observed by Hayabusa, Science 312, 1330 (2006)

24. B.J. Gladman, F. Migliorini, A. Morbidelli, V. Zappalà, P. Michel, A. Cellino, Ch. Froeschlé, H.F. Levison, M. Bailey, and M. Duncan: Dynamical lifetimes of objetcs injected into asteroid belt resonances, Science 277, 197 (1997)

25. B.J. Gladman, P. Michel, and Ch. Froeschlé: The Near-Earth Object Population, Icarus 146, 176 (2000)

26. R. Greenberg, W.F. Bottke, and M. Nolan: Collisional and Dynamical History of Ida, Icarus 120, 106 (1996)

27. K.A. Holsapple: The Scaling of Impact Processes in Planetary Sciences, Annu. Rev. Earth Planet. Sci. 21, 333 (1993)

28. K. Holsapple, I. Giblin, K. Housen, A. Nakamura, and E. Ryan: Asteroid Impacts: Laboratory Experiments and Scaling Laws, In: Asteroids III, eds. by W. F. Bottke Jr, A. Cellino, P. Paolicchi, and R. P. Binzel (Univ. of Arizona Press, Tucson, 2002) pp. 443-462.

29. K.R. Housen, R.M. Shumidt, and K.A. Holsapple: Crater Ejecta Scaling Laws Fundamental Forms Based on Dimensional Analysis, J. Geophys. Res. 88, 2485 (1983)

30. H.H. Hshieh, and D. Jewitt: A Population of Comets in the Main Asteroid Belt, Science 312, 561 (2006)

31. M. Kaasalainen, J. Durech, B.D. Warner, Y.N. Krugly, N.M. Gaftonyuk: Acceleration of the rotation of asteroid 1862 Apollo by radiation torques, Nature 446 , $420(2007)$

32. T. Kadono, J. Kameda, K. Saruwatari, H. Tanaka, S. Yamamoto, and A. Fujiwara: Surface roughness of alumina fragments caused by hypervelocity impact, Planet. Space Sci. 54, 212 (2006)

33. Y. Kozai: Kiyotsugu Hirayama and His Families of Asteroids In: Proc. Seventyfive (75) years of Hirayama asteroid families: The role of collisions in the solar system history, eds by Y Kozai, R.P. Binzel, and T. Hirayama, Astronomical Society of the Pacific Conference Series 63, 1 (1994)

34. S.C. Lowry, A. Fitzsimmons, P. Pravec, D. Vokrouhlický, H. Boehnhardt, P.A. Taylor, J.-L. Margot, A. Galád, M. Irwin, J. Irwin, and P. Kusnirák: Direct Detection of the Asteroidal YORP Effect, Science 316, 272 (2007)

35. J.S. Mathis, W. Rumple, and K.H. Nordsieck: The size distribution of interstellar grains, Astrophysical J. 217, 425 (1977) 
36. A.S. McEwen, J.M. Moore, and E.M. Shoemaker: The Phanerozoic Impact Cratering Rate: Evidence from the Farside of the Moon, J. Geophys. Res. 102, 9231 (1997)

37. K. Marti and T. Graf: Cosmic-Ray Exposure History of Ordinary Chondrites, Annu. Rev. Earth Planet. Sci. 20, 221 (1992)

38. H.J. Melosh: Impact Cratering A Geologic Process, Oxford University Press, New York (1989)

39. P. Michel, W. Benz, and D. C. Richardson: Modelling Collisions Between Asteroids: From Laboratory Experiments to Numerical Simulations: Disruption of Fragmented Parent Bodies as the Origin of Asteroid Families, Nature 421, 608 (2003)

40. T. Michikami, A. M. Nakamura, N. Hirata, R.W. Gaskell, R. Nakamura, T. Honda, C. Honda, K. Hiraoka, J. Saito, H. Demura, M. Ishiguro, and H. Miyamoto: Size-frequency statistics of boulders on global surface of asteroid 25143 Itokawa, Earth, Planet. Space, in press (2007)

41. A. Milani and Z. Knezevic: Asteroid proper elements and the dynamical structure of the asteroid belt, Icarus 107, 219 (1994)

42. A. Morbidelli and B.J. Gladman: Orbital and temporal distributions of meteorites originating in the asteroid belt, Meteoritics and Planetary Science 33, 999 (1998)

43. A. Morbidelli, W. F. Bottke Jr., Ch. Froeschle, and P. Michel: Origin and Evolution of Near-Earth Objects, In: Asteroids III, eds. by W. F. Bottke Jr, A. Cellino, P. Paolicchi, and R. P. Binzel (Univ. of Arizona Press, Tucson, 2002) pp. 409-422.

44. A. Nakamura and A. Fujiwara: Velocity Distribution of Fragments Formed in a Simulated Collisional Disruption, Icarus 92, 132 (1991)

45. A.M. Nakamura: Laboratory Studies on the Velocity of Fragments from Impact Disruptions, The Institute of Space and Aeronautical Science Report, 651 (1993)

46. A.M. Nakamura, A. Fujiwara, T. Kadono: Velocity of finer fragments from impact, Planet Spacs Sci. 42, 1043 (1994)

47. A.M. Nakamura, P. Michel, and M. Setoh: Weibull Parameters of Yakuno Basalt Targets Used in Documented High-Velocity Impact Experiments, J. Geophys. Res. (Planets) 112, 10.1029/2006JE002757 (2007)

48. A.M. Nakamura, T. Michikami, N. Hirata, A. Fujiwara, R. Nakamura, M. Ishiguro, H. Miyamoto, H. Demura, K. Hiraoka, T. Honda, C. Honda, J. Saito, T. Hashimoto, and T. Kubota, Impact Process of Boulders on the Surface of Asteroid 25143 Itokawa- Fragments from Collisional Disruption, Earth, Planet. Space, in press (2007)

49. S. Nakazawa, S. Watanabe, M. Kato, Y. Iijima, T. Kobayashi, and T. Sekine: Hugoniot equation of state of basalt, Planet. Space Sci. 45, 1489 (1997)

50. S. Nakazawa, S. Watanabe, Y. Iijima, and M. Kato: Experimental Investigation of Shock Wave Attenuation in Basalt, Icarus 156, 539 (2002)

51. D. Nesvorný, W.F. Bottke Jr., L. Dones, and H.F. Levison: The recent breakup of an asteroid in the main-belt region, Nature 417, 720 (2002)

52. D.P. O'Brien, and R. Greenberg: The collisional and dynamical evolution of the main-belt and NEA size distributions, Icarus 178, 179 (2005)

53. T. Okada, K. Shirai, Y. Yamamoto, T. Arai, K. Ogawa, K. Hosono, and M. Kato:X-ray Fluorescence Spectrometry of Asteroid Itokawa by Hayabusa, Science 312, 1338 (2006)

To be published in: "Small Bodies in Planetary Systems". (Mann, Nakamura, Mukai eds.) 
54. P. Paolicchi, S. Marchi, D. Nesvorny, et al.: Towards a general model of space weathering of S-complex asteroids and ordinary chondrites, Astron. Astrophys. 464, 1139 (2007)

55. C.A. Polanskey and T.J. Ahrens: Impact spallation experiments - Fracture patterns and spall velocities, Icarus 87, 140 (1990)

56. D.C. Richardson, Z.M. Leinhardt, H.J. Melosh, W.F. Bottke Jr., and E. Asphaug.: Gravitational Aggregates: Evidence and Evolution, In: Asteroids III, eds. by W.F. Bottke Jr, A. Cellino, P. Paolicchi, and R.P. Binzel (Univ. of Arizona Press, Tucson, 2002) pp. 501-515.

57. E. Ryshkewitch: Compression Strength of porous Sintered Alumina and Zirconia, J. Am. Ceram. Soc., 36, [2], 65 (1953)

58. A.S. Rivkin, E.S. Howell, F. Vilas, and L.A. Lebofsky: Hydrated Minerals on Asteroids: The Astronomical Report, In: Asteroids III, eds. by W.F. Bottke Jr, A. Cellino, P. Paolicchi, and R.P. Binzel, (Univ. of Arizona Press, Tucson, 2002), pp. 235-253.

59. J. Saito, H. Miyamoto, R. Nakamura, M. Ishiguro, T. Michikami, A.M. Nakamura, H. Demura, S. Sasaki, N. Hirata, C. Honda, A. Yamamoto, Y. Yokota, T. Fuse, F. Yoshida, D.J. Tholen, R.W. Gaskell, T. Hashimoto, T. Kubota, Y. Higuchi, T. Nakamura, P. Smith, K. Hiraoka, T. Honda, S. Kobayashi, M. Furuya, N. Matsumoto, E. Nemoto, A. Yukishita, K. Kitazato, B. Dermawan, A. Sogame, J. Terazono, C. Shinohara, H. Akiyama: Detailed Images of Asteroid 25143 Itokawa from Hayabusa, Science 312, 1341 (2006)

60. P. Spurný, J. Oberst, and D. Heinlein: Photographic observations of Neuschwanstein, a second meteorite from the orbit of the Príbram chondrite, Nature 423, 151 (2003)

61. Y. Takagi, H. Mizutani, and S.-I. Kawakami: Impact fragmentation experiments of basalts and pyrophyllites, Icarus 59, 462 (1984)

62. D.J. Tholen and M.A. Barucci: Asteroid Taxonomy In: Asteroids II, eds. by R. P. Binzel, T. Gehrels, and M. S. Matthews (Univ. of Arizona Press, Tucson, 1989) pp. 298-315.

63. J.I. Trombka, S.W. Squyres, J. Bruckner, W.V. Boynton, R.C. Reedy, T.J. McCoy, P. Gorenstein, L.G. Evans, J.R. Arnold, R.D. Starr, L.R. Nittler, M.E. Murphy, I. Mikheeva, R.L. McNutt, T.P. McClanahan, E. McCartney, J.O. Goldsten, R.E. Gold, S.R. Floyd, P.E. Clark, T.H. Burbine, J.S. Bhangoo, S.H. Bailey, M. Petaev: The Elemental Composition of Asteroids 433 Eros: Results of the NEAR-Shoemaker X-ray Spectrometer Science 289, 2101 (2000)

64. J. Veverka, P. Thomas, A. Harch, B. Clark, J.F. Bell, B. Carcich, J. Joseph, S. Murchie, N. Izenberg, C. Chapman, W. Merline, M. Malin, L. McFadden, M. Robinson: NEAR Encounter with Asteroid 253 Mathilde: Overview Icarus 140, 3 (1999)

65. J. Veverka, M. Robinson, P. Thomas, S. Murchie, J.F. Bell, N. Izenberg, C. Chapman, A. Harch, M. Bell, B. Carcich, A. Cheng, B. Clark, D. Domingue, D. Dunham, R. Farquhar, M.J. Gaffey, E. Hawkins, J. Joseph, R. Kirk, H. Li, P. Lucey, M. Malin, P. Martin, L. McFadden, W.J. Merline, J.K. Miller, W.M. Owen, C. Peterson, L. Prockter, J. Warren, D. Wellnitz, B.G. Williams, D.K. Yeomans: NEAR at Eros: Imaging and Spectral Results, Science 289, 2088 (2000)

66. Weibull, W.: A Statistical Theory of the Strength of Materials Ingvetensk. Akad Handl. 151, 1 (1939)

67. M. Yanagisawa and T. Itoi: Impact Fragmentation Experiments of Porous and Weak Targets. In: Proc. of Seventy-five (75) years of Hirayama asteroid families:

To be published in: "Small Bodies in Planetary Systems". (Mann, Nakamura, Mukai eds.) 
The role of collisions in the solar system history, eds. by Y. Kozai, R.P. Binzel, and T. Hirayama, Astronomical Society of the Pacific Conference Series 63, 243 (1994).

68. D.K. Yeomans, J.-P. Barriot, D.W. Dunham, R.W. Farquhar, J.D. Giorgini, C.E. Helfrich, A.S. Konopliv, J.V. McAdams, J.K. Miller, W.M. Owen, Jr., D.J. Scheeres, S.P. Synnott, and B.G. Williams: Estimating the Mass of Asteroid 253 Mathilde from Tracking Data during the NEAR Flyby, Science 278, 2106 (1997)

69. K. Yomogida and T. Matsui: Physical properties of ordinary chondrites, J. Geophys. Res. 88, B11 9513 (1983)

70. V. Zappalà, A. Cellino, A. Dell'Oro: Physical and Dynamical Properties of Asteroid Families, In: Asteroids III, eds. by W. F. Bottke Jr, A. Cellino, P. Paolicchi, and R. P. Binzel (Univ. of Arizona Press, Tucson, 2002) pp. 619-631.

To be published in: "Small Bodies in Planetary Systems". (Mann, Nakamura, Mukai eds.) 
To be published in: "Small Bodies in Planetary Systems".

(Mann, Nakamura, Mukai eds.)

Submitted to Lecture Notes in Physics series by Springer Funded by the 21the Century COE Program "Origin and Evolution of Planetary Systems" 


\section{Index}

$Q^{*}, 18,19$

albedo, 5, 6

albedo, geometric, 5, 6, 7

asteroid family, 1, 4, 20

Asteroid:243 Ida, 1, 7, 8, 20, 21

Asteroid:25143 Itokawa, 2, 3, 4, 7, 8, 20, $21,22,23,24$

Asteroid:253 Mathilde, 7, 8, 20, 21

Asteroid:433 Eros, 1, 7, 8, 21

Asteroid:951 Gaspra, 1, 20

catastrophic disruption, 1

Centaurs, 4

chondrite, $7,20,21$

chondrite, carbonaceous, 7,12

chondrite, ordinary, 6, 7, 10, 12, 14, 21

cosmic ray exposure age, $\mathrm{CRE}$ age, 10

density, 1, 2, 7, 8, 10, 12, 13, 15, 17, 18, $19,20,21,24$

Galileo, 20

Hayabusa, 2, 3, 21

IDP, 1

Kuiper belt, 2

Kuiper belt objects, 2, 4, 24 main asteroid belt, MB, 1, 2, 3, 4, 6, 7, $8,9,11,18,20$

mean motion resonance, $3,4,5$ meteorite, 1, 2, 6, 7, 9, 10, 12, 14, 15

NEAR, 7, 20, 21

near Earth object, NEO, 3, 4, 19, 21

near-Earth asteroids, NEA, 14

porosity, $2,7,8,13,15,19,20,21$

secular resonance, 3,5

shock wave, $8,10,12,17,21$

size distribution, 3

size distribution, asteroids, 5, 6, 9

size distribution, boulders, 23

size distribution, dust, 19

size distribution, fragments, 19, 20, 23

space weathering, 6, 7, 10, 20

strength, 11, 13, 14, 15, 16, 17, 18, 19, 22,23

taxonomic class, 1

trans-Neptunian object, TNO, 4

Weibull distribution, 15, 16

Weibull parameter, 13, 15, 16

Yarkovsky effect, 4, 5, 9, 10, 24

YORP effect, 24 